\title{
Iridoschisis: high frequency ultrasound imaging. Evidence for a genetic defect?
}

Mt Sinai School of Medicine, New York, USA

J Danias

J W Eichenbaum

D Z Reinstein

Cornell University Medical College, New York, USA

I M Aslanides

R H Silverman

D J Coleman

Correspondence to:

D Jackson Coleman, MD, Department of

Ophthalmology, Cornell

University Medical College,

1300 York Avenue, Room

A-855, New York, NY

10021 , USA.

Accepted for publication 26 August 1996

\author{
John Danias, Ioannis M Aslanides, Joseph W Eichenbaum, Ronald H Silverman, Dan Z \\ Reinstein, D Jackson Coleman
}

\begin{abstract}
Aims-To elucidate changes in the anatomy of the anterior chamber associated with iridoschisis, a rare form of iris atrophy, and their potential contribution to angle closure glaucoma.

Methods-Both eyes of a 71-year-old woman with bilateral iridoschisis and fibrous dysplasia and her asymptomatic 50-year-old daughter were scanned with a very high frequency $(50 \mathrm{MHz})$ ultrasound system.

Results-The symptomatic patient exhibited diffuse changes in the iris stoma with an intact posterior iris pigmented layer in both eyes. These changes were clinically compatible with the lack of iris transillumination defects. Additionally, iris bowing with a resultant narrowing of the angle occurred. The asymptomatic daughter showed discrete, but less severe iris stromal changes.

Conclusion-This is the first detailed study of high frequency ultrasonic imaging of the iris in iridoschisis. The observed structural changes suggest angle narrowing by forward bowing of the anterior iris stroma may be a mechanism of IOP elevation in this condition. The ultrasonic detection of iris changes in the asymptomatic daughter of the symptomatic patient and the association of iridoschisis with fibrous dysplasia suggest a possible genetic component in the pathogenesis of this condition.
\end{abstract}

(Br f Ophthalmol 1996;80:1063-1067)

Iridoschisis is a rare condition with approximately 100 cases being reported in the literature to date. An association of iridoschisis with angle closure glaucoma has been established. ${ }^{1}$ Also an association with interstitial keratitis has been suggested. ${ }^{2}$

We describe a case of iridoschisis associated with fibrous dysplasia and nanophthalmos. In an attempt to elucidate the relation of glaucoma and iridoschisis, high frequency ultrasound (HFU) images of the patient's irides were obtained. This is the first detailed report of HFU imaging of the iris in iridoschisis (a single HFU image of the iris in iridoschisis appears in a textbook ${ }^{3}$ ). In addition, HFU imaging of the patient's daughter was also obtained to determine whether subclinical changes were present, thus implicating possible genetic factors in the pathogenesis of this rare condition.

\section{Methods}

Both patients received a complete ophthalmic examination including refraction, slit-lamp examination, gonioscopy, and dilated fundus examination.

The patients were scanned with a high frequency ultrasound system of our own design. ${ }^{45}$ The system utilised a polyvinylidene fluoride (PVDF) transducer with a nominal centre frequency of $50 \mathrm{MHz}$. The transducer was mounted onto a motion control assembly consisting of two stepper motors $(10 \mu \mathrm{m}$ step size) aligned at right angles to each other. Scan sequences were performed under computer control. Each sequence consisted of a series of parallel, rectilinear scans in either sagittal or transverse planes focusing on the iris and angle structures at various clock hours. Echo data were digitised at a sample rate of $200 \mathrm{MHz}$ and stored on hard disk. The number of scans per sequence, the interval between scan planes and the lateral width of each scan were all programmable.

Scanning was performed using an immersion technique. The supine patient received one drop of proxymetacaine (proparacaine) hydrochloride $0.5 \%$ and the lids were retracted with a Barraquer lid speculum. A plastic drape with a central aperture and an adhesive backing was then attached to the skin in the periorbital area to form a watertight seal. Sterile normal saline was then added to form a waterbath approximately $2 \mathrm{~cm}$ in depth above the globe. The transducer (focal length 12 $\mathrm{mm}$ ) was then lowered into the waterbath and aligned so as to place the area of interest (the iris) in the focal plane. The use of a waterbath allowed non-contact ultrasonic examination. A fixation light was used to help direct and maintain steady gaze during scanning. Each scan sequence consisted of 16 slices $8 \mathrm{~mm}$ in depth by $10 \mathrm{~mm}$ in width with an interscan interval of $0.25 \mathrm{~mm}$.

\section{Results}

FG is a 71-year-old Hispanic female with a history of excision of fibrous dysplasia of the left frontal bone and superior orbital decompression in 1979. The patient was first seen in 1969 after being referred by an optometrist for 

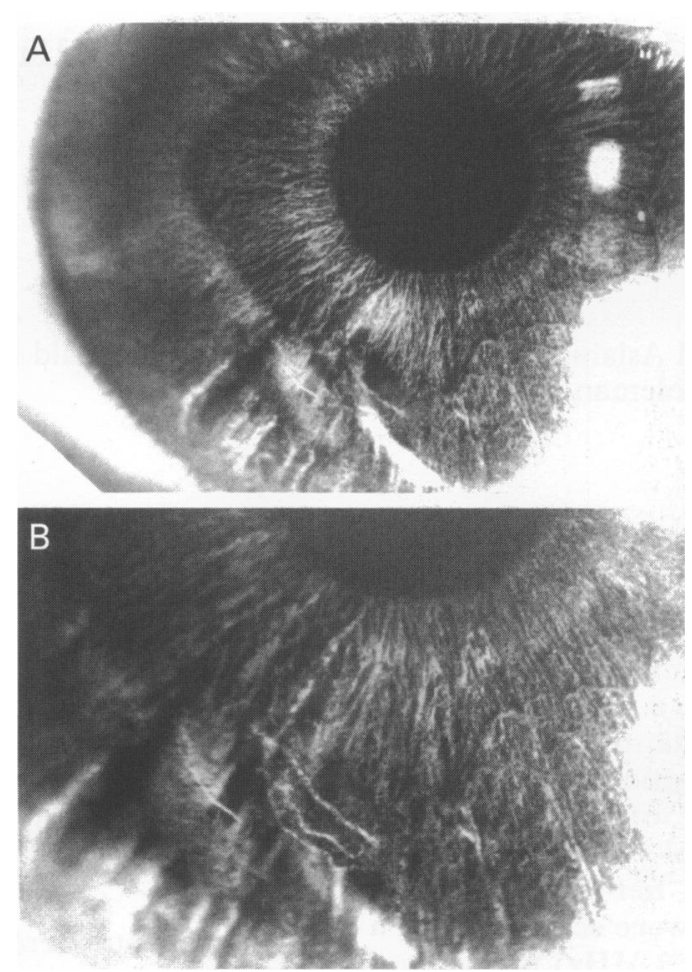

Figure 1 Slit-lamp photographs of the iris of patient FG (left eye). Typical changes of iridoschisis are seen in all quadrants $(A)$ but are more prominent inferiorly, seen at a higher magnification (B).

elevated intraocular pressure (IOP). On that particular visit and in all subsequent ones, her IOP was always found to be below $19 \mathrm{~mm} \mathrm{Hg}$ in both eyes. Angle structures and iris anomalies had not been recorded.

On 21 November 1994, her best corrected visual acuity was $20 / 25$ in the right eye and $20 / 40$ in the left with $+1.75 \mathrm{D}$ and $+0.75 \mathrm{D}$ in right and left eyes respectively. Slit-lamp examination was remarkable for changes in the irides of both eyes (Fig 1). The irides showed areas of anterior stromal rarefaction with a 'shredded wheat' appearance. The iris strands were attached to the peripheral iris stroma and pupillary margin but not in the mid-peripheral region. A few loose iris stromal fibres were noted floating in the anterior chamber. All quadrants were affected, with changes being more pronounced inferiorly and in the left eye. The pupils were both reactive and round. An area of touch between iris stromal strands and the corneal endothelium was present inferiorly in the right eye; however, the cornea was clear.

On gonioscopy, angle structures were visible only with eccentric positioning of the Zeiss lens (steep approach). In the right eye, the angle was graded $(+2) \times 270^{\circ}$ and slit $\times 90^{\circ}$ temporally. In the left eye, the angle was graded as slit $\times 180^{\circ}$ inferiorly and superiorly and (+1) $\times 180^{\circ}$ nasally and temporally. Indentation gonioscopy revealed angle structures to the ciliary body $(+4) \times 360^{\circ}$ in the right eye and to the scleral spur $(+3) \times 360^{\circ}$ in the left. Both eyes were nanophthalmic with corneal diameters of $9.5 \mathrm{~mm}$ and axial lengths on A-scan of $21.77 \mathrm{~mm}$ (right eye) and $22.14 \mathrm{~mm}$ (left eye). IOP was recorded at 23 and $26 \mathrm{~mm} \mathrm{Hg}$ in the right and left eye respectively. In the left eye, a hyaloid remnant was noted arising from the disc. Optic cup to disc ratios were 0.5 and 0.8

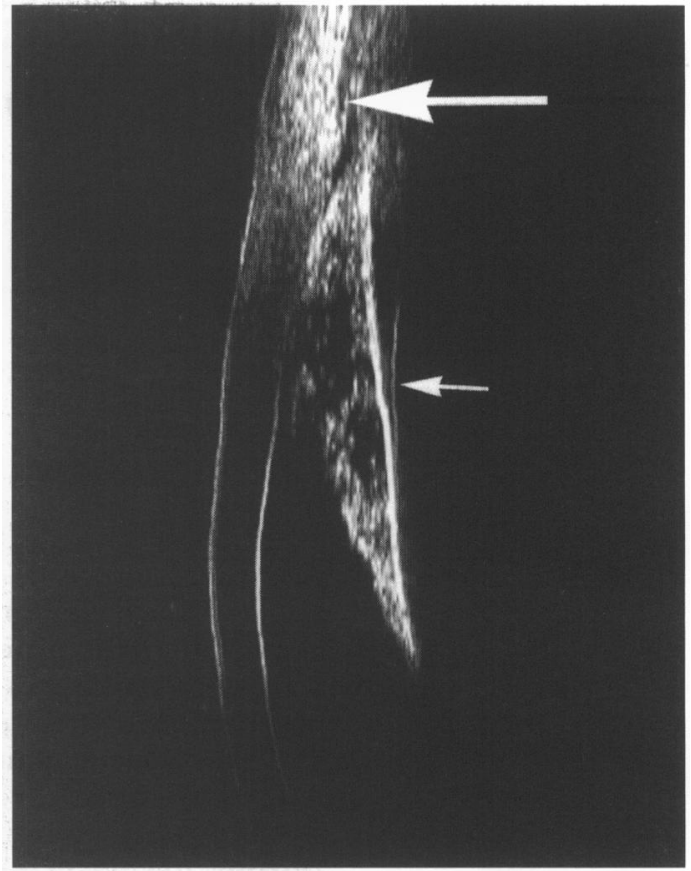

Figure 2 High frequency ultrasound image of the iris of patient FG (left eye inferiorly). The posterior pigment epithelium is intact and can be seen draping over the anterior capsule (small arrow). Extensive stromal rarefaction can be observed mid-peripherally with deep iris crypts extending to the posterior pigmented epithelium. The anterior stromal fibres bow forward, effectively obstructing the angle (large arrow). An area of iridocorneal touch can be observed.

right and left eye, respectively. Goldmann perimetry revealed full fields in both eyes although the Humphrey hemifield in the left eye was found to be borderline glaucomatous. No stigmata of congenital syphilis were noted.

The patient underwent successful laser iridectomy in the left eye without complications. After laser iridectomy, IOPs were initially $19 \mathrm{~mm} \mathrm{Hg}$ right eye and $15 \mathrm{~mm} \mathrm{Hg}$ left eye (day 1). Angle structures became visible in the treated eye. Gonioscopy revealed angle structures graded $(+2) \times 180^{\circ}$ nasally and inferiorly and $(+1) \times$ $180^{\circ}$ superiorly and temporally. Identation gonioscopy revealed angle structures to $(+4) \times 180^{\circ}$ inferiorly and nasally and to $(+3) \times 180^{\circ}$ superiorly and temporally. However, 1 month after laser iridectomy, IOP was recorded to be $23 \mathrm{~mm} \mathrm{Hg}$ in the left eye with essentially unchanged gonioscopy findings.

One month after laser iridectomy, HFU imaging was obtained in an effort to understand the mechanism of elevation of IOP. HFU images demonstrated the absence of central iris stroma in both eyes (Figs 2-4). The amount of stromal rarefaction was unequal in various areas of the iris (Fig 2), being greatest inferiorly in the left eye, as expected from clinical examination (Figs 2 and 3). The posterior pigmented epithelium of the iris was intact (Figs $2-4$ ), in sharp contrast with the overlying stroma. The anterior iris strands were visualised to extend from the iris periphery to the sphincter although not adhering to the underlying stroma (Figs 2-4). Deep iris crypts extending to the posterior pigment epithelium were seen in many areas (Figs 2 and 3 ).

The area of iridocorneal touch in the left eye is visualised in Figure 2. As can be observed in 


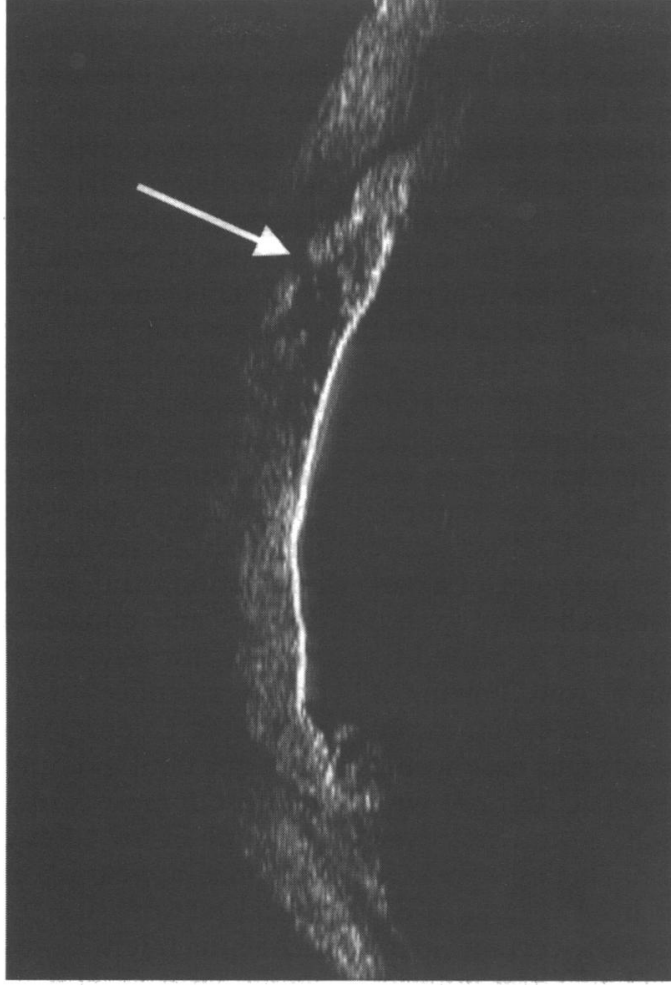

Figure 3 High frequency ultrasound image of the iris periphery of patient FG (left eye inferiorly). Deep iris crypts can be seen (arrow). The posterior pigment epithelium appears to be flaccid. The angle is very narrow.

Figures 2-4, the angle was narrow; however, in certain areas it definitely remained open in both eyes. The posterior pigment epithelium was noted to have a slight anterior bowing in all quadrants in both eyes; this is slightly more pronounced in the right eye (Fig 4). In the left eye the posterior pigment epithelium seems to be more flaccid, draping over the anterior lens surface (Figs 2 and 3 ).

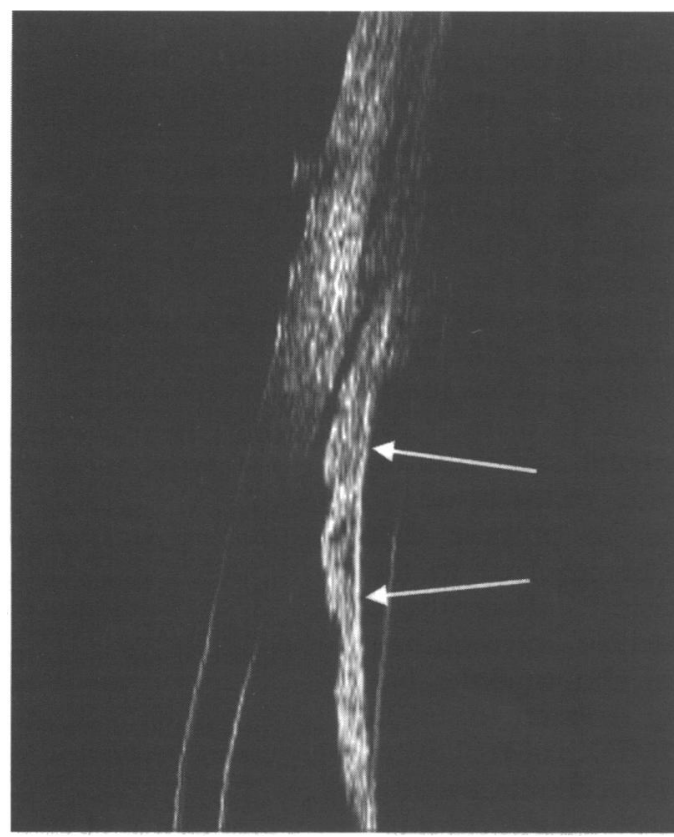

Figure 4 High frequency ultrasound image of the iris of patient FG (right eye inferiorly). Stromal defects are present but are not as prominent as in the left eye. Note the posterior pigmented epithelial layer bowing anteriorly (arrows). The angle is narrow.

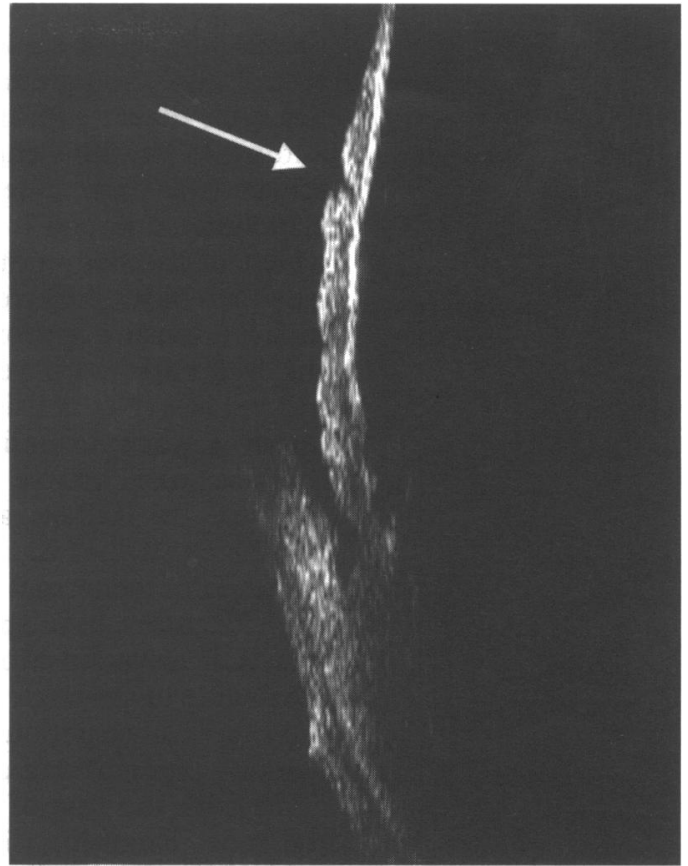

Figure 5 High frequency ultrasound image of the iris of the daughter of FG (left eye). A deep crypt can be seen extending to the posterior pigmented epithelium (arrow). The posterior epithelium is intact. Angle is open, although somewhat narrow.

$\mathrm{AH}$ is the 50-year-old daughter of FG. After her mother's diagnosis of angle closure glaucoma and iridoschisis she was called in for full ophthalmic evaluation. Horizontal corneal diameters were $10 \mathrm{~mm}$ in both eyes. Angles were graded $(+2) \times 270^{\circ}$ and $(+4) \times 90^{\circ}$ in both eyes opening to $(+4) \times 360^{\circ}$ with pressure. IOP was $21 \mathrm{~mm} \mathrm{Hg}$ in both eyes. Cup to disc ratio was 0.3 in both eyes. The irides of the daughter did not show iridoschisis; however, prominent radial ridges of the iris were seen. HFU imaging revealed normal posterior pigment epithelium corroborating the lack of transillumination defects on clinical examination (Fig 5). The iris stroma showed some rarefaction (Fig 5) compared with the appearance of a normal iris. Deep crypts extending to the posterior pigmented layer were observed (Fig 5).

\section{Discussion}

Iridoschisis has been associated with angle closure glaucoma. ${ }^{1}$ However, although angle closure glaucoma is relatively common, iridoschisis is rare. In addition, of the cases reported to date only approximately $50 \%$ had angle closure glaucoma. ${ }^{16-9}$ It has been proposed that avascular necrosis of the iris stroma in patients with acutely elevated IOP may lead to iridoschisis. ${ }^{19}$ Such a view is supported by evidence of hyalinisation of the anterior stromal vessels and dissolution of the vessels in the deep stromal layers near the dilator muscle. ${ }^{10}$ However, others argue that the stromal changes are unlikely to be related to ischaemia." Iris fluorescein angiography has shown that only radial stromal vessels of various calibres are filled, but not the connecting vessels or capillaries. ${ }^{12}$ In contrast, in essential iris atrophy with hole formation, the holes were 
surrounded by ischaemic zones with leaking vessels at the periphery of the ischaemic areas. ${ }^{12}$ Also, no significant vascular abnormalities were detected in iridoschisis by the above authors. ${ }^{11}$ In our symptomatic patient, iridoschisis was associated with angle closure glaucoma, although it is difficult to determine which of the two entities preceded the other. The patient was referred for evaluation of ocular hypertension approximately 25 years before she was noted to have the typical changes of iridoschisis. No consistent elevation of IOP, however, was substantiated until recently.

The clinical course of our patient supports the view that IOP elevation is not due to pupillary block. If that was the case IOP would be expected to be normal in the presence of a patent iridectomy. In the past it has been proposed that IOP elevation in iridoschisis is caused by narrowing of the angle from forward bowing of the iris ${ }^{1}$ or obstruction of the trabecular meshwork by pigment and debris. ${ }^{11}$ Our case would agree with the first of the above: the eye with the most extensive iridoschisis and narrower angle had the higher IOP. Angles appeared narrow in all quadrants in both eyes on HFU imaging (Figs 2-4) although certain areas remained open. It appeared that the anterior iris stroma was bowed forward in excess of the posterior pigment epithelium, effectively obstructing the angle. The partial bowing of the posterior pigment epithelium might be attributed to loss of the structural integrity of the iris. The more flaccid appearance of this layer in the eye that had undergone laser iridotomy supports this view. These observations suggest that the elevation of IOP in this case is due to narrowing of the angle by the bowed anterior leaflet of the iris, although clogging of the trabecular meshwork with debris cannot be excluded as a secondary mechanism.

Our patient was bilaterally nanophthalmic with iridoschisis being more severe in the smaller eye. A hyaloid remnant was also present in this eye. A similar association of iridoschisis with microphthalmos has been reported. ${ }^{1314}$

The patient described above also suffered from fibrous dysplasia involving the frontal bone. Although these two rare conditions (iridoschisis and fibrous dysplasia) can occur together by mere chance, it is also possible that they arise because of a common underlying defect in mesodermal maturation as has previously been suggested for both of them. ${ }^{15}{ }^{16}$ In the past, other developmental abnormalities have been associated with iridoschisis. ${ }^{7117}$ This has prompted some to propose that hereditary factors might be involved in the pathogenesis of iridoschisis. ${ }^{18-20}$ Recently, a case of a child with iridoschisis, microphthalmia, and other congenital abnormalities has been reported with an $\mathrm{Xp}$ deletion (distal to DXS16 and proximal to DXS143). ${ }^{21}$ Also, other reported cases of iridoschisis in patients with polyploidy seem to involve the $\mathrm{X}$ chromosome. ${ }^{22}$ HFU imaging of the iris in both the mother and daughter reported here supports the possibility of a role for a genetic factor in the pathogenesis of iridoschisis. Although the daughter (21 years younger) did not have clinical evidence of iridoschisis, HFU imaging shows early changes of the iris stroma suggestive of iridoschisis. It remains to be seen if she will develop clinically evident iridoschisis in the future. A similar case of both mother and daughter affected by iridoschisis has been reported in the past. ${ }^{23}$

What seems most confusing about iridoschisis is that although a degenerative process could explain many of the reported cases, others cannot be explained on this basis. It seems that part of the problem arises from overlapping clinical pictures of iridoschisis with other conditions. ${ }^{6}$ These include essential iris atrophy and glaucomatous atrophy for the older population, and mesodermal dysgenesis syndromes and congenital hypoplasia of the iris stroma for the younger patients. ${ }^{611}$ It is, however, conceivable that iridoschisis might represent a common clinical picture of more than one clinical entity. Thus, it is possible that a defect in mesodermal maturation is causative for some of the cases of iridoschisis (especially the ones appearing at a younger age), while other factors like trauma, IOP spikes, or ischaemia might be responsible for some of the senile cases. It is equally possible that a rare genetic defect predisposes certain patients to develop a particular form of iris atrophy when subjected to various exogenous or endogenous insults.

\section{Conclusion}

In this report we document anatomical changes in the iris of a symptomatic mother having iridoschisis and her asymptomatic daughter. These changes consisted of stromal rarefaction, forward bowing of the anterior layers of the iris, and an intact posterior pigmented layer in the mother and stromal changes in the daughter. The above observations suggest that the forward bowing of the anterior stromal tissue may cause angle narrowing with resultant elevation of IOP. In addition, the less extensive changes observed in the daughter hint at the presence of a genetic component in the aetiology of this condition.

This work was supported in part by PHS grant EY03183 (to DJC), Research to Prevent Blindness, Inc New York, and the Dyson Foundation, New York, USA.

Dr I M Aslanides is a post-doctoral fellow of Fight for SightPrevent Blindness America.

1 Salmon JF, Murray ADN. The association of iridoschisis and primary angle closure glaucoma. Eye 1992;6:267-72.

2 Salvador F, Linares F, Merita I, Amen M. Unilateral iridoschisis associated with syphilitic interstitial keratitis and schisis associated with syphilitic interstitial
glaucoma. Ann Ophthalmol 1993;25:328-9.

3 Ritch R, Lowe RF. Angle closure glaucoma: clinical types. In: Ritch R, Shields MB, Krupin T, eds. The glaucomas. 2nd ed. New York: Mosby-Year Book, 1996:830.

4 Reinstein DZ, Silverman RH, Rondeau MJ, Coleman DJ Epithelial and corneal thickness measurements by highfrequency ultrasound digital signal processing. Ophthalmology 1994;101:140-6.

5 Aslanides IM, Libre PE, Silverman RH, Reinstein DZ, Lazzaro DR, Rondeau MJ, et al. HFU imaging in pupillary block. Br ₹ Ophthalmol 1995;79:972-6.

6 Duke-Elder S, Perkins ES. Diseases of the uveal tract. In: Duke-Elder S, ed. System of ophthalmology, St Louis: CV Mosby, 1958; Vol IX:694-8.

7 Romano A, Treister G, Barishack R, Stein R. Iridoschisis and angle closure glaucoma. Ophthalmologica 1972;164:199-207. angle closure glaucoma. Ophthalmologica 1972,164:199-207. atlas. New York: Harper and Row, 1989:606-7.

9 Mills PV. Iridoschisis. Br $₹$ Ophthalmol 1967;51:158-64. 
10 Albers EC, Klein BA. Iridoschisis. A clinical and histopathologic study. Am f Ophthalmol 1958;46:794.

11 Rodrigues MC, Spaeth GL, Krachmer JH, Laibson PR Iridoschisis associated with glaucoma and bullous keratopathy. Am $\mathcal{F}$ Ophthalmol 1983;95:73-91.

12 Vannas A. Fluorescein angiography of the vessels of the iris in pseudoexfoliation of the lens capsule, capsular glaucoma and some other forms of glaucoma. Acta Ophthalmol 1969; 105 (supl):

13 Summers CG, Doughman DJ, Letson RD, Lufkin M. Juvenile iridoschisis and microphthalmos. Am $f$ Ophthalmol

14 Johnson MR, Bachynski BN. Juvenile iridoschisis and microphthalmos. Am $\mathcal{F}$ Ophthalmol 1986;101:742-3.

15 Carter LF. Progressive senile fibrillar atrophy of the iri stroma (iridoschisis). Am $\mathcal{f}$ Ophthalmol 1953;36:967-9.

16 Jakobiec FA, Jones IS. Mesenchymal and fibro-osseous tumors. In: Tasman W, ed. Duane's clinical ophthalmology.
Philadelphia: JB L ippincott, 1994; vol 12, Chapter 44, 26-30. 17 Remlein-Mozolewska G. Przypadek iridoschisis u 10-letnie dziewczynki. Klin Oczna 1973;43:185-7.

18 Bonnet M, Vianey E. Contribution a l' etude de l'iridoschisis. Arch Ophthalmol 1970;30:129-34

19 Seidler-Dymitrowska M, Dzierzykraj-Rogalski T. Iridoschisis rzadkie schorzenie teczowski. Klin Oczna 1954;24: 207-10.

20 Posner A, Gorin G. Iridoschisis and glaucoma. Am $\mathcal{f}$ Ophthalmol 1958;45:451.

21 Thies U, Gopal Rao VVN, Engel W, Schmidtke J. Physical mapping of two Xp markers DXS16 and DXS143. Hum Genet 1991;86:418-20.

22 Hanicka M, Kleezkowska A, Makowska J, Sokolowski J, Jarzyk K. Rozszczep teczowki u dziewczynki z kariotypem 48, XXXX. Poll Tyg Lek 1969;24:1164-9.

23 Streif EB, Faggioni R, Scouras J. Iridoschisis familial complique de cornea guttae. Ophthalmologica 1974;169:411-5. 